\title{
La agencia como \\ forma organizativa de \\ optimización de servicios \\ públicos. Pasado, presente \\ y futuro en una España \\ en crisis
}

Alejandra Botol

\section{RESUMEN}

En los actuales tiempos de crisis globalizada es esencial plantear la organización administrativa en términos de eficiencia, eficacia y racionalidad. Redimensionar el sector público es prioritario, y para ello se busca una ingeniería organizativa capaz de optimizar el control del gasto y la prestación de servicios esenciales. Este trabajo analiza el potencial de la forma de agencia para ello, en particular desde la perspectiva de las agencias estatales españolas. El estudio comienza prestando atención al fenómeno polimórfico y polisémico de la agencia en abstracto, con una perspectiva de derecho comparado, para pasar después a analizar el bagaje concreto de las agencias estatales en España y su situación presente y futura.

1 Doctora en Derecho y catedrática de la Universidad de Oviedo, Oviedo, España. Su actividad de investigación cubre los temas de organización administrativa y la prestación y derechos de los usuarios de servicios públicos. Entre sus trabajos se encuentra $L a$ Administración instrumental en el proceso (Madrid: Reus, 2011) y El enigma de la descentralización funcional (Barcelona: Erasmus, 2013), así como contribuciones a obras publicadas en diferentes países. Correo-e: botoalejandra@uniovi.es. Fecha de recepción: 10 de diciembre de 2014. Fecha de modificación: 20 de febrero de 2015. Fecha de aceptación: 3 de abril de 2015. Para citar el artículo: Boto, A. (2015). La agencia como forma organizativa de optimización de servicios públicos. Pasado, presente y futuro en una España en crisis. Revista Digital de Derecho Administrativo n. ${ }^{\circ}$ 13, Universidad Externado de Colombia, pp. 45-61. DOI: $10.18601 / 21452946 . n 13.05$ 
Palabras clave: derecho administrativo; derecho comparado; agencia simplificación y modernización administrativa ${ }_{i}$ autoridades administrativas independientes.

\title{
Agency as an Organizational Choice for Public Services Optimization. Past, Present and Future in a Spain in Crisis
}

\author{
ABSTRACT
}

In the current global crises situation, it is essential to contemplate the administrative organization in terms of efficiency, effectiveness and rationality. A remodeling of the public sector is imperative and to do so requires a type of organizational engineering, which is able to optimize control over public expenses and the provision of essential services. This work looks at an agency's potential for such a mission, and particularly employs the perspective of the Spanish State Agencies. This paper begins by abstractly addressing the polymorphic and polysemic phenomenon of agencies from a Comparative Law perspective and then moves on to analyze the specific background of state agencies in Spain and their present and future situation.

Keywords: Administrative Law; Comparative Law; Agency; Administrative Simplification and Modernization, Independent Agencies.

\section{INTRODUCCIÓN}

Las páginas que siguen forman parte de las labores de difusión de los avances parciales del proyecto denominado "Modelos jurídico-organizativos de optimización de las prestaciones esenciales del Estado de bienestar" que, bajo la dirección del profesor Leopoldo Tolivar Alas y con la financiación del Ministerio español de Economía y Competitividad ${ }^{2}$, se lleva a cabo en la Universidad de Oviedo por parte del grupo de investigación SPAG (Servicios públicos, Administraciones y garantías). El fin de tal proyecto es indagar, a partir del análisis doctrinal, legislativo y jurisprudencial, histórico y de derecho comparado, sobre los modelos que mejor pueden conseguir optimizar las prestaciones esenciales del Estado de bienestar ante los ajustes estructurales que la coyuntura económica actual exige. 
Dicha situación socioeconómica, que a pesar de ser global ha golpeado a países como España con una singular intensidad, evidencia la urgente necesidad de replantear la organización administrativa en términos de economía y efectividad. Con todo, ni los términos ni las necesidades son por completo novedosos, pues como los ciclos económicos, también las oleadas de reforma administrativa tienden a repetirse. Y en tal contexto se torna claramente pertinente el estudio del bagaje y prospectiva de las agencias estatales, introducidas en España en virtud de una Ley de 2006, que precisamente las ligaba a la optimización en la prestación de servicios.

El análisis parte de considerar los perfiles difusos de la agencia en abstracto, como forma organizativa de origen anglosajón y con proyección internacional pero recepcionada con dificultades en el mundo latino, estudiando en particular el caso de Francia e Italia. Se pasa después a considerar sus perfiles más concretos, a raíz de la legislación española de 2006, para terminar reflexionado sobre el impacto de la crisis económica y el futuro inminente.

El trabajo toma en cuenta únicamente la escala estatal dentro del Estado compuesto español por razones ligadas al ámbito de aplicación de la Ley de 2006; no obstante, no se evitan algunas referencias útiles e ilustrativas a lo acaecido en tiempos recientes en los ámbitos autonómico y local.

La perspectiva y metodología es la propia del derecho administrativo, toda vez que resulta ya indiscutible que lo organizativo en la Administración, aunque linde con otras muchas disciplinas, es algo jurídico (TACCOGNA, 2014, pp. 1-12).

\section{LA AGENCIA: FENÓMENO POLIMÓRFICO Y POLISÉMICO. REFLEXIONES DE DERECHO COMPARADO}

Salvo quizás en los casos en que se afronta el estudio de una concreta institución sectorial muy bien delimitada, la importación de términos propios del derecho comparado no es nunca un fenómeno neutral, y menos en cuestiones de organización (MOCKLE, 2006, p. 135). Así, al hablar en términos genéricos de "agencias" es inevitable evocar la experiencia del New Public Management y las desconcentradas executive agencies Next Steps del Reino Unido en los años 80, o de su derivación más moderna, las independent regulatory agencies americanas.

Ciertamente, existe una tendencia convergente, al menos entre los países miembros de la $O C D E$, que hace que este tipo de entes se multipliquen hasta un punto tal que incluso se ha llegado a catalogarlos como "accesorios de moda" (POLLiTT et al., 2001, p. 286). La convergencia, con todo, no es perfecta, porque las diferentes idiosincrasias y especialidades de los Estados siguen estando siempre presentes, de modo que muchas veces la tendencia a la homogeneidad, aun real, es perturbadoramente superficial (MOYNIHAN, 2006) y 
puede conducir fácilmente al error, sobre todo si no se tiene en consideración la tradición jurídica propia.

En los países del entorno español, y aunque las realidades concretas que se agrupan bajo la denominación de agencia son muy variopintas (ZWART y VERHEY, 2003), la figura suele estar presidida por las ideas de eficacia, autonomía y flexibilidad, y su proliferación ligada a razones de modernización, de gobernanza, de descentralización, de aumento de la eficacia, y en general de optimización de la gestión administrativa, que pasa a estar orientada hacia los ciudadanos; así ocurre sin ir más lejos tanto en Italia (CORSI, 2005) como en Francia (GUETTIER, 2006). El mismo fenómeno se ha experimentado también en América Latina (Prats i CaTALÀ, 2005).

$Y$ sin embargo es preciso ser cautelosos, pues muchas veces aparentes similitudes esconden en realidad diferencias importantes cuyo análisis, precisamente por darse en contextos en apariencia próximos, puede ser de lo más revelador. En principio, una aproximación superficial al tema parece conducir, en efecto, a la conclusión de que todos estos sistemas están profundamente emparentados, aunque solo sea por el uso de una terminología común, donde la traducción parece hacerse casi innecesaria (agenzie-agences-agencias). No obstante, no podemos dejar que la proximidad geográfica y lingüística, el bagaje común de nuestra tradición histórica o las similitudes del contexto económicosocial nos lleven a dar por válidas unas conclusiones precipitadas. Más allá de las apariencias se hace enseguida patente que los términos se utilizan, en uno y otro caso, para referirse a realidades sustanciales tremendamente distintas entre sí, con lo que la misión se complica, el esfuerzo adquiere más valor y el estudio se enriquece, porque es cierto que existen importantes similitudes, pero también no menos relevantes diferencias.

En Italia, por ejemplo, son "agencias" en sentido estricto las creadas en virtud del Decreto Legislativo 300/1999 (art. 8) como entes de carácter técnicoorganizativo sin personalidad jurídica, régimen al que se han incorporado de forma reciente la Agenzia per le infrastrutture stradali ed autostradale (Decreto Ley 98/2011, art. 36) o la Agenzia per l'Italia digitale (Decreto Ley 83/2012, art. 19), pero también lo son las agencias fiscales con personalidad jurídica propia del mismo Decreto Legislativo 300/1999 (art. 57). En un punto intermedio (TACCOGNA, 2014, pp. 186-191) se encuentran entes como la Agenzia italiana per il farmaco (Decreto Ley 269/2003, art. 43) o la Agenzia per la coesione territoriale (Decreto Ley 101/2013, art. 10). No son agencias, en cambio, pese a llevar tal denominación formal, la Agenzia nazionale per l'attrazione degli investimenti e lo sviluppo d'impresa (Ley 296/2006, art. 1.460), que es una sociedad pública por acciones; la Agenzia per la rappresentaza negoziale delle pubbliche amministrazioni (Decreto Legislativo 165/2001, art. 40), ente público con personalidad jurídica; o la Agenzia nazionale per la regolazione e la vigilanza in materia di acqua (Decreto Ley 70/2011, art. 10), que vendría a ser una autoridad independiente. 
En relación con este último ejemplo, en cierta medida es lógico que surjan interrogantes en la frontera entre agenzie y autorità, pues ambas son formas organizativas presididas por una fuerte indeterminación conceptual ${ }_{i}$ figuras heterogéneas que han conocido un auge reciente, proliferando en poco tiempo de la mano de influencias extranjeras y coincidiendo en los mismos momentos cronológicos. Ni unas ni otras son, por separado, categorías unitarias, por lo que es fácil que sus distintos subtipos puedan, en la práctica, solaparse entre sí. La particular adaptación alla italiana de los modelos extranjeros de referencia (Next Steps, Quangos y Regulatory Agencies, sobre todo), la utilización de terminología equívoca, la incertidumbre, o incluso la carencia de una regulación general, y la prolífica y discordante literatura sobre la materia tampoco han contribuido a simplificar el enfoque.

Así las cosas, pues, puede decirse que agencias y autoridades independientes son dos de las innovaciones que más han contribuido a la metamorfosis reciente de la realidad administrativa en Italia (DURET, 2003). No es este un fenómeno aislado, sino que sigue una tendencia globalizada, que liga perfectamente con lo ocurrido en Francia.

También en el país galo, en efecto, puede apreciarse una tremenda heterogeneidad de estatutos jurídicos bajo la etiqueta general de "agencias" (MAHERZI, 2010, pp. 89-90) y la confusión entre la terminología de agencia y la naturaleza de autoridad independiente es evidente en el caso, por ejemplo, de la Agence française de lutte contre le dopage (Ley 405/2006, art. 2). Por otra parte, tal y como ya he tenido la ocasión de analizar en otro lugar (BOTO ÁLVAREZ, 2014a), de forma muy reciente ha mutado la denominación de la tradicional Agence d'évaluation de la recherche et de l'enseignement supérieur, que ha dejado de ser formalmente una "agencia", pasando a ser un "Alto Consejo"; un giro no exento de cierto maquillaje y refuerzo de su independencia (Ley 660/2013, art. 90).

Ante ello, puede llegar a pensarse que en realidad las agencias no son sino un paso intermedio en la evolución hacia el policentrismo administrativo que culmina finalmente con la instauración de las autoridades independientes (PIAZZA, 1999, p. 7) o, al contrario, que la evolución y el desarrollo de las distintas nuevas fórmulas de organización administrativa de los últimos tiempos (agencias, autoridades, etc.) puede ser potencialmente reagrupable en un único modelo general, el de las agency (NAPOLITANO, 2014, pp. 30-32); y todo esto más allá de las calificaciones usadas en cada momento para denominar a cada figura. Predicar la necesidad de una racionalización de las categorías sería a estas alturas no solo repetitivo, sino hasta cierto punto también ilusorio ${ }_{i}$ no en vano la flexibilidad y el particularismo están en la base misma de la razón de ser de las agencias y de las autoridades independientes, tal y como ya analizara el Consejo de Estado francés (Les autorités administratives indépendantes - Rapport public, 2001). Ahora bien, a quien sí puede pedírsele con fundamento un esfuerzo de racionalización y análisis es al legislador que instituye los entes en concreto. 
Tal reflexión es también pertinente en España, donde desde mucho antes de la aprobación de la Ley 28/2006, que introdujo normativamente en nuestro país la figura de la agencia, ya existían organismos que adoptaban tal denominación formal aunque respondían en realidad a otras tipologías jurídicas. Así, la actual Agencia de Protección de Datos (Ley Orgánica 15/1999, art. 135), a pesar de su denominación "esconde" una verdadera autoridad independiente, mientras que la importantísima Agencia Estatal de la Administración Tributaria fue concebida en realidad como entidad de derecho público con régimen jurídico propio (Ley 31/1990, art. 103). Particular en sumo grado fue la efímera Agencia Industrial del Estado (Ley 5/1996, art. 2), en funcionamiento durante apenas un año bajo la forma de ente de derecho público, que sin embargo actuaba sujeto al derecho privado. Existen, incluso después de 2006, otras "agencias" nominales que responden en realidad al modelo de organismo autónomo tradicional de la Ley 6/1997: es el caso de la Agencia de Información y Control Alimentarios (Ley 12/2013, Disposición Adicional primera) o la Agencia Española de Consumo, Seguridad Alimentaria y Nutrición (Real Decreto 19/2014, art. 1). De forma análoga, la conocida Agencia EFE es, a pesar de su denominación ambigua, una sociedad anónima, adscrita a la Sociedad Estatal de Participaciones Industriales desde 2001. Por último encontramos también meros órganos con designación equívoca, como la Agencia Nacional de Evaluación y Prospectiva, en la Secretaría General de Ciencia, Tecnología e Innovación de la Secretaría de Estado de Investigación, Desarrollo e Innovación, del Ministerio de Economía y Competitividad, o la Agencia de Calidad del Sistema Nacional de Salud (Ley 16/2003, art. 60).

Si se considerase además el ámbito autonómico, la confusión aumentaría de forma exponencial (FONT i LLOVET, 2008, pp. 748-750; ÁlVAREZ CARREÑO, 2009, pp. 14-15). Lo mismo ocurre cuando se tiene en cuenta el ordenamiento de la Unión Europea, porque una de las formas de gestión de los programas comunitarios es precisamente la gestión "por redes de Agencias Nacionales", como forma de delegación, por parte de la Comisión, de responsabilidades de ejecución a entidades nacionales investidas de una misión de servicio público que colaboran en la aplicación de determinadas políticas comunitarias (Comunicación de la Comisión al Consejo y al Parlamento Europeo de 13 de noviembre de 2001). Igualmente, y solo por citar otro ejemplo de confusión terminológica entre agencias y autoridades independientes, desde 2009 la Agencia de Cooperación de los Reguladores de la Energía ejerce, a escala comunitaria, las tareas de las autoridades reguladoras de los Estados miembros (Reglamento (CE) n. ${ }^{\circ}$ 713/2009 del Parlamento Europeo y del Consejo).

Pero bien, volvamos a 2006 y a la aprobación en España de la primera regulación material sobre agencias que, como se verá, aparece formalmente ligada a la mejora de la prestación de los servicios públicos. 


\section{LA LEY 28/2006, DE 18 DE JULIO, DE AGENCIAS ESTATALES PARA LA MEJORA DE LOS SERVICIOS PÚBLICOS}

Con fecha 19 de julio de 2006 se publicó en el Boletín Oficial del Estado la Ley 28/2006, de 18 de julio, de Agencias Estatales para la mejora de los servicios públicos. Con ella, y tras el estandarte de la modernización de las Administraciones y de la búsqueda de la calidad y la eficiencia, se creaba una nueva fórmula organizativa, la de la agencia, con la renovada esperanza, tal y como se establece en la Exposición de Motivos de la ley, de solucionar las rigideces y limitaciones que habían hecho fracasar a los modelos organizativos anteriores. Las agencias no se conciben, pues, como figuras atípicas con un contenido específico, sino como una nueva categoría típica de la Administración instrumental, con vocación técnica, además, de ser la única en el futuro.

La nueva ley no deroga el sistema tradicional, en puridad solo añade un nuevo tipo de organismo público al sistema bipartito existente hasta el momento ${ }^{3}$; no obstante, prevé que los organismos existentes se acaben transformando en agencias y que, a partir de su entrada en vigor, las agencias sean la forma habitual de creación de nuevas organizaciones instrumentales en la Administración General del Estado.

La Ley de Agencias Estatales no contiene mención expresa alguna a su ámbito de aplicación, ni al título competencial en función del que se dicta. Sin embargo, desde su misma Exposición de Motivos ya se aprecia que es una norma que viene a complementar el sistema preexistente y, por lo tanto, que ha de compartir su mismo planteamiento (SALVADOR ArMENDÁRIZ, 2007, p. 404).

La filosofía latente detrás de esta nueva fórmula de ingeniería organizativa pública es la preocupación por garantizar la autonomía y la flexibilidad en la gestión de las formas organizativas instrumentales de la Administración, potenciando la planificación, la transparencia y la evaluación por resultados.

3 El sistema está recogido en la Ley 6/1997, sobre organización y funcionamiento de la Administración General del Estado, que en materia de entes instrumentales distingue entre personificaciones jurídico-públicas, a las que engloba en conjunto en la categoría de organismos públicos, y personas jurídicas que adoptan formas jurídico-privadas: las sociedades mercantiles. Los organismos públicos se definen como entidades de derecho público que actúan como organizaciones instrumentales de la Administración General del Estado, dependientes de ella (art. 1), a los que se encomiendan tareas de ejecución o gestión tanto administrativas de fomento o prestación como de contenido económico (art. 2.3) en régimen de descentralización funcional (art. 41). Los organismos públicos se dividen a su vez en organismos autónomos y entidades públicas empresariales. Ambos son de naturaleza pública, pero difieren en cuanto a su régimen jurídico. Así, los primeros siempre se van a regir por el derecho administrativo (art. 45), mientras que las segundas se van a regir, en muchos aspectos, por el derecho privado y solo en algunos concretos por el derecho administrativo (art. 53). 
En su versión original, la parte dispositiva de la ley contaba con treinta y un artículos, que se completan con nueve importantísimas disposiciones adicionales y dos finales ${ }^{4}$. Las agencias estatales se definen como entidades de derecho público, dotadas de personalidad jurídica y patrimonio propio, con autonomía de gestión y capacidad para ejercer potestades públicas. La pluricitada ley insiste a lo largo de todo su articulado en subrayar su autonomía en la gestión, y en el control y la responsabilidad por los resultados de su actuación. Su naturaleza ${ }^{5}$ se establece en términos amplísimos en el artículo 2 de la ley, que dispone que las agencias son creadas para el cumplimiento de los programas correspondientes a las políticas públicas que desarrolla la Administración estatal. Una formulación especialmente vaga y difusa, alrededor de la cual la doctrina comenzó en seguida a discutir. A título personal, creo que la definición del ámbito funcional de las agencias es un aspecto sobre el que el legislador quiso pasar intencionadamente de puntillas. De ahí que no exista rúbrica literal sobre sus funciones, y de ahí también su enunciación confusa e incidental. No debe obviarse que esta ley forma parte de un paquete de reformas normativas para la modernización de la Administración estatal (con distintas vertientes de preocupación tales como la igualdad, el empleo público, las nuevas tecnologías, etc.); una filosofía de conjunto que no puede dejar de ser considerada. A nivel conceptual, la Ley de Agencias aporta más bien poco a la configuración de la arquitectura institucional teórica de dicha Administración, es en la dimensión práctica donde sus reformas inciden (mejora de la eficiencia, planificación de objetivos, evaluación del desempeño del personal, controles financieros, etc.). Así, las agencias no son más que una "vestimenta", una nueva "etiqueta" para los organismos públicos estatales pero que, en sí misma, como categoría, presenta un carácter difuso, diluido y hasta casi podría decirse, inexistente. Parece que el legislador se centra más en las cuestiones operativas internas que en las vertientes, por así decirlo, macro organizativas. Todo ello porque en el fondo la Ley de Agencias no es sino una herramienta para conseguir un fin: crear el entramado institucional necesario para conseguir el objetivo final de la reforma: la tan traída y llevada modernización administrativa ${ }^{6}$.

Las agencias se crean con la aprobación de sus estatutos a través de real decreto. Eso sí, necesariamente antes tiene que existir una autorización por

4 En 2007 se añadiría una nueva disposición adicional, relativa al régimen fiscal de las agencias en el impuesto sobre sociedades, modificada después en 2011.

5 Es la redacción literal de la ley, si bien sería más correcto hablar aquí de "funciones" (Martín-Caro García, Bettschen Capa y Estoa PéreZ, 2007, pp. 37-38).

6 Debe recordarse, en esta línea, que el propio gobierno impulsor de la ley reconocía que uno de los objetivos centrales que con ella se perseguía era la puesta en marcha de una agencia concreta: se trataba de la Agencia de Evaluación de las Políticas Públicas y la Calidad de los Servicios (disposición adicional primera), que fue definida incluso como "la otra pata del proyecto de Ley" y que se juzga, de hecho, como el paso imprescindible para poder implantar una nueva cultura de gestión de los servicios públicos (GARDE ROCA, 2005). 
1 ley ${ }^{7}$. En su régimen jurídico se someten a las reglas establecidas en su estatuto, y supletoriamente se les aplicarán también las disposiciones de las normas de organización general y sectorial que les sean aplicables (CosCULLuELA MONTANER, 2006, pp. 41 ss.).

A los aspectos de la organización interna de las agencias se dedica todo el capítulo segundo de la ley ene studio, donde se hace referencia a los órganos de gobierno (Presidente y Consejo Rector), ejecutivos (Director) y de control interno. Con prolijidad se detalla el papel del ministerio de adscripción en el funcionamiento de la agencia, algo que ha sido visto por la doctrina como una forma de remarcar el vínculo de instrumentalidad que existe entre ellos. Otra manifestación de la relación de instrumentalidad se ha visto en el contrato de gestión, instrumento jurídico que pretende garantizar específicamente la "gestión transparente por objetivos" y que ha sido valorado por la doctrina como la principal novedad de la citada ley. Es un instrumento plurianual, que aunque se llame "contrato" viene a ser más bien una programación de objetivos y medios; se complementa con un plan de acción anual.

Las disposiciones adicionales de la LAE tienen una envergadura fuera de lo común, que está en relación con la orientación "utilitarista" que el legislador hace, como ya se ha comentado, del entramado institucional creado por la parte dispositiva de la ley y al que hacíamos referencia más arriba al hablar del ámbito funcional de las agencias. Así la disposición adicional quinta prevé la transformación de los organismos públicos existentes en agencias, a través de un real decreto del Consejo de Ministros y sin mencionar que sea necesaria autorización parlamentaria alguna, aunque en teoría antes de proceder a la transformación se deberá, conforme a la disposición adicional novena, enviar un informe al Parlamento. Por su parte, la disposición adicional sexta establece la previsión general de que todos los organismos que se creen en un futuro adopten la forma de agencia estatal.

Contenido importantísimo es el de la disposición adicional séptima, que fija las relaciones con las Cortes Generales y que ha sido actualizada en virtud de la Ley 22/2013, de 23 de diciembre, relativa a la Oficina Presupuestaria de estas. Así se prevé que el Presidente de la agencia y el Ministro de adscripción comparezcan ante las Comisiones de las Cámaras para informar de los aspectos de la gestión de la agencia para los que sean requeridos. Asimismo, también tendrán que remitir los correspondientes informes anuales sobre su actividad.

En las tres primeras disposiciones adicionales se contiene, por último, la autorización para la creación de lo que parecen doce nuevas agencias, entre ellas, evidentemente, está incluida la Agencia de Evaluación que, como ya se

7 Este aspecto fue uno de los más polémicos en la tramitación parlamentaria de la ley (PÉREZ GÁLVEZ, 2007, pp. 195 ss.). 
ha apuntado, fue uno de los ejes centrales de la reforma. Decimos que "parecen" doce nuevas agencias porque en algunos casos ya existía un organismo público con el mismo nombre, con lo cual más parece que estemos ante una transformación (mecanismo al que se refiere la disposición adicional quinta y que no parece requerir de autorización parlamentaria) que ante la verdadera autorización legislativa para la creación. Por cierto que el régimen de la inicialmente prevista como Agencia Estatal de Seguridad del Transporte Terrestre ha sido actualizado en virtud del Real Decreto-ley 1/2014, de 24 de enero, de reforma en materia de infraestructuras y transporte, y otras medidas económicas, que modifica el nombre y las competencias de lo que habrá de ser la futura Agencia Estatal de Seguridad Ferroviaria, para dar cumplimiento a los requerimientos de la Comisión Europea, sobre independencia del organismo de seguridad ferroviaria.

La confusión entre los mecanismos de creación y de transformación está servida, y a mayor abundamiento debe apuntarse que, fuera de la Ley de 2006, existen también previsiones de transformación o de creación de agencias en algunas leyes sectoriales, y no siempre con la corrección sistemática que sería deseable. Así, por ejemplo, la Ley 5/2007, de 3 de abril, de la Red de Parques Nacionales, cuya derogación parece por otra parte inminente, autorizó en su disposición adicional segunda la creación de una Agencia Estatal Red de Parques Nacionales, sin matizar qué es lo que ocurriría llegado el momento de su puesta en funcionamiento con el Organismo Autónomo Parques Nacionales en su configuración actual. Lo mismo ocurre en la Ley Orgánica de Universidades 6/2001, de 21 de diciembre, cuyo artículo 32, tras la reforma operada por Ley Orgánica 4/2007, de 12 de abril, autoriza la creación de la Agencia Nacional de Evaluación de la Calidad y la Acreditación, remitiendo de forma expresa al texto de la Ley 28/2006, sin precisar qué es lo que ocurrirá con la fundación que hasta ahora, y en virtud de lo establecido en la redacción originaria del susodicho artículo, realiza las funciones que se encomiendan a la agencia estatal y que porta la misma denominación (la fundación ANECA). Mientras tanto, en un supuesto muy similar, la Ley 55/2007, de 28 de diciembre, del Cine, opta por una técnica radicalmente distinta, "autorizando" la transformación del existente Organismo Autónomo Instituto de la Cinematografía y de las Artes Audiovisuales en Agencia Estatal de la Cinematografía y de las Artes Audiovisuales en su disposición adicional primera, algo que parece en principio una disposición superflua habida cuenta de la previsión general de la disposición adicional quinta de la Ley de Agencias. Finalmente, la Ley 27/2011, de 1 de agosto, sobre actualización, adecuación y modernización del sistema de Seguridad Social, en su disposición adicional séptima, ordena la creación de la Agencia de la Administración de la Seguridad Social con una integración orgánica de las entidades gestoras y los servicios comunes. La integración anunciada se aventura polémica ya que no es completa, pues no afecta a todos los entes gestores, y los parámetros jurídicos de la Ley 26/2006 
ofrecen potenciales dificultades de acoplamiento con el régimen actual ( $\mathrm{SA}_{\mathrm{A}}$ RAGOSSÀ i SARAGOSSÀ, 2012).

Un supuesto de creación ex novo de una agencia estatal se contiene en la disposición adicional duodécima de la Ley 14/2011, de 1 de junio, de la Ciencia, la Tecnología y la Innovación, en relación con la Agencia Estatal para la Investigación.

A pesar de todas estas previsiones, muy poco es lo que se ha avanzado en estas transformaciones por razones a las que la crisis económica no es ajena, como seguidamente se verá, y que no son exclusivas del contexto español (CASSESE, 2014).

\section{LA IRRUPCIÓN DE LA CRISIS ECONÓMICA}

Como ya se ha explicado, la Ley 28/2006 prevé que la forma de agencia estatal sea la configuración de los organismos públicos estatales que se creen en el futuro (disposición adicional sexta), y que los ya existentes se reconduzcan también a dicha forma (disposición adicional quinta). El planteamiento parece absoluto y rotundo, pero en realidad tampoco lo es tanto.

Para empezar, porque la propia disposición adicional quinta, en su apartado primero, condiciona la transformación de los existentes organismos públicos en agencias a que exista identidad entre los objetivos y actividades de unos y la naturaleza de las otras; además el plazo para la transformación expiraba en julio de 2008 y aquel momento llegó sin que se hubieran producido avances masivos. Tan solo se habían creado siete agencias estatales, todas de las ya autorizadas en las disposiciones adicionales de la propia Ley de 2006. Eran: la Agencia Estatal de Evaluación de las Políticas Públicas y la Calidad de los Servicios (cuyos estatutos se aprobaron por Real Decreto 1418/2006, de 1 de diciembre), completamente de nuevo cuño; la Agencia Española de Cooperación Internacional para el Desarrollo (aprobados sus estatutos por Real Decreto 1403/2007, de 26 de octubre), que sustituyó a la precedente Agencia Española de Cooperación Internacional que tenía configuración de organismo autónomo, la Agencia Estatal del Boletín Oficial del Estado (estatutos aprobados por Real Decreto 1495/2007, de 12 de noviembre), que se subrogó en la totalidad de bienes, derechos y obligaciones del existente Organismo Autónomo BOE; la Agencia Estatal del Consejo Superior de Investigaciones Científicas (Real Decreto 1730/2007, de 21 de diciembre), que sustituyó al Organismo Autónomo Consejo Superior de Investigaciones Científicas; la de Seguridad Aérea (Real Decreto 184/2008, de 8 de febrero), que no es heredera de ningún organismo público anterior sino que asume funciones que correspondían a la Dirección General de Aviación Civil; la Agencia Antidopaje (Real Decreto 185/2008, de 8 de febrero), también de nuevo cuño, y la Agencia de Meteorología (Real Decreto 186/2008, de 8 de febrero), que sucede a la Dirección General del 
Instituto Nacional de Meteorología del Ministerio de Medioambiente, ahora por primera vez con personalidad jurídica propia, puesto que tal Instituto, heredero a su vez del Instituto Central Meteorológico, había pasado por diferentes denominaciones y adscripciones pero formalmente nunca se había constituido como organismo público diferenciado.

La crisis socioeconómica global comenzaría por aquel entonces a cebarse en España, y los efectos sobre la pretendida transformación organizativa no se hicieron esperar. Así, en el punto segundo de la disposición adicional trigésimo novena de la Ley $2 / 2008$, de 23 de diciembre, de presupuestos para 2009, se estableció, tras la ampliación en un año más del plazo para proceder a la transformación, que la creación de nuevas agencias debería ir en todo caso precedida de un informe preceptivo y vinculante del Ministerio de Economía y Hacienda que, evaluando los efectos de dicha creación sobre el gasto público, determinase la oportunidad de su creación. Además, también se establecía un límite máximo: las agencias estatales que se creasen en total durante el ejercicio 2009 no podrían exceder, en ningún caso, de cinco. Eran los comienzos de los ajustes de las cuentas públicas.

Habiendo resultado tal límite amplio en exceso, y persistiendo la situación de crisis, la Ley de presupuestos para 2010 reiteró las mismas previsiones, con el límite absoluto de cuatro nuevas agencias (disposición adicional trigésima primera de la Ley 26/2009, de 23 de diciembre), si bien el Real Decreto-Ley $8 / 2010$, de 20 de mayo, por el que se adoptaron medidas extraordinarias para la reducción del déficit público, en su disposición adicional quinta suspendió tal previsión para establecer que en 2010 no se autorizaría la creación de ninguna nueva agencia estatal.

La misma regla se prorrogó para 2011 en virtud de la disposición adicional vigésima sexta de la Ley 39/2010, de 22 de diciembre. Ese año vería empero la luz la Agencia Estatal Agencia Española de Medicamentos y Productos Sanitarios (estatutos aprobados por Real Decreto 1275/2011), sucesora del anterior organismo autónomo homónimo al amparo de una autorización presupuestaria expresamente contenida en el apartado dos de la misma disposición adicional vigésima sexta de la Ley 39/2010 para la creación de agencias en aquellos supuestos en que con anterioridad a la entrada en vigor del Real Decreto-Ley 8/2010 se hubiera emitido, conjuntamente por los Ministerios de la Presidencia y de Economía y Hacienda, informe favorable conforme a la disposición adicional trigésima primera de la Ley 26/2009, de 23 de diciembre, de Presupuestos Generales del Estado para el año 2010.

Las limitaciones a la creación de agencias continuarían en 2012 por mor de la disposición adicional décima séptima de la Ley 2/2012, de 29 de junio (con la excepción de la Agencia Estatal para la Investigación, ya presentada, que se habría de realizar sin aumento de gasto público, y no sufragarse con créditos del presupuesto financiero del Estado), y para 2013, con la misma 
excepción, por obra de la disposición adicional octogésima séptima de la Ley 17/2012, de 27 de diciembre.

La restricción sigue vigente aún para 2014, conforme a la disposición adicional octogésima sexta de la Ley 22/2013, de 23 de diciembre, manteniendo la misma excepción sobre la Agencia Estatal para la Investigación y añadiendo la de la Agencia Estatal de Seguridad Ferroviaria, también más arriba presentada. La segunda no aparece mencionada en la primera versión del Proyecto de Ley de presupuestos para 2015, que solo considera como excepción a la prohibición de crear agencias estatales en 2015 la de la Agencia para la Investigación en el texto de la disposición sexagésima octava de la norma que en el momento de escribir estas páginas se encuentra en tramitación.

En todo caso, de ninguna de ellas se tiene noticia a día de hoy, como tampoco nada se sabe sobre la eventual transformación de otros organismos públicos ya existentes en agencias: ni de aquella general que debería iniciarse a instancia del Gobierno, ni de aquella otra más particular auspiciada por leyes especiales como la Ley de la Red de Parques Nacionales, la Ley del Cine o la Ley sobre actualización, adecuación y modernización del sistema de Seguridad Social.

\section{CONCLUSIONES}

Como se ha visto, a pesar de la revolución de la estructura organizativa de la Administración que se esperaba con la entrada en vigor de la Ley de Agencias en el año 2006, hasta el momento tan solo existen en España operativas ocho agencias estatales. La crisis económica es un factor a considerar en tal fracaso, pero no es el único.

Así, el propio título de la Ley de Agencias inducía de forma un tanto pretenciosa a la confusión, pues ciertamente con la sola reforma organizativa no puede pretenderse mejorar automáticamente la calidad de los servicios públicos, máxime cuando las agencias estatales no están llamadas a ejercer funciones prestacionales de forma directa. Como mucho, tal efecto podría haberse predicado tan solo de la Agencia de Evaluación de las Políticas Públicas y Calidad de los Servicios (COSCulluela MONTANER, 2006, p. 29), o tendría que haberse matizado que la idea de "servicio público" se entendía a los efectos de aquella ley como la mera ejecución de programas correspondientes a políticas públicas de competencia del Estado (Pérez GÁlveZ, 2006, pp. 528-545).

En definitiva, con la misma elección del término "agencia" el legislador español estaba haciéndose abanderado del valor supremo, cargado axiológicamente de connotaciones positivas, de una modernización administrativa largamente esperada, pero por otra parte indefinida. De ahí que se realicen también grandilocuentes declaraciones formales, sobre todo en la Exposición de Motivos de la ley, sobre la flexibilidad, autonomía y racionalización de la 
nueva (y en teoría definitiva) forma organizativa de nuestra Administración instrumental. Sin embargo, en el pasado ya se ha demostrado que tales declaraciones teóricas son insuficientes y que la racionalización y la uniformidad en la Administración institucional española parecen una utopía. De la misma forma, iniciar un verdadero proceso de agencification al estilo del derecho comparado requiere de una reforma de mucho mayor calado.

En todo caso, al menos de momento y a causa de la crisis económica, el proceso de cambio de paradigmas organizativos con las agencias estatales por bandera ha quedado frenado. No han faltado voces, empero, que han apuntado el interés de la figura para su traspaso a las escalas autonómica (RoDRíGuEZArana Muñoz y Sendín García, 2014) y local (Prieto Romero, 2010, pp. 335-336).

La tendencia, sin embargo, va en dirección contraria, hacia la supresión y fusión de entes, en un movimiento cuya última causa es la crisis económica y que, de nuevo, no es exclusivo del contexto español, también se da de forma intensa en Italia, donde se ha llegado a hablar incluso de una maniobra tagliaenti (TORIELLO, 2014, pp. 10-26).

Volviendo a España, sucede lo mismo de forma evidente en el caso de las comunidades autónomas, comprometidas con la estabilidad presupuestaria y el control del gasto público a raíz del Acuerdo 1/2010, de 22 de marzo, del Consejo de Política Fiscal y Financiera, por el que se aprobó el Acuerdo marco con las Comunidades Autónomas y Ciudades con Estatuto de Autonomía sobre sostenibilidad de las finanzas públicas para el período 2010-2013, intensificado tras el Acuerdo 5/2012, de 17 de enero, del Consejo de Política Fiscal y Financiera, por el que se adoptaron compromisos en materia de reordenación y racionalización del sector público instrumental autonómico y de control, eficiencia y reducción del gasto público gestionado por el mismo (RAmOs CARBAJAL, 2013). Para su seguimiento se han elaborado informes periódicos sobre los planes puestos en marcha por las distintas comunidades autónomas para dar cumplimiento a los compromisos de supresión, transformación o creación de entes, incluyendo posibles fusiones e integraciones, así como, en su caso, la enajenación de participaciones, e informes sobre el Inventario de Entes dependientes de las comunidades autónomas. En ellos se aportan datos que arrojan un resultado de ahorros estimados por el conjunto de medidas de reestructuración en las comunidades autónomas de 4.042.533,42 miles de euros, derivados tanto de medidas de extinción de entes como de otras medidas de racionalización de sus sectores públicos instrumentales.

Finalmente, en el ámbito local, ha visto la luz de forma reciente la reforma acometida por la Ley 27/2013 en la Ley de Bases de Régimen Local de 1985, provocando un redimensionamiento del sector público local fuertemente cuestionado en su racionalidad y sostenibilidad. Y es que, entre nosotros, la preocupación por la configuración organizativa de la Administración local siempre se ha vinculado más a cuestiones cuantitativas de tipología y de 
planta de la realidad de nuestras entidades locales (AlmEIDA CeRREDA, 2013, pp. 66-68) que a un estudio específico sobre la dinámica de las formas de lo público (Palomar Olmeda, 2010: 42). El legislador de 2013 ha seguido esta misma línea y así, a pesar de la notable importancia que se da formalmente a las cuestiones de organización administrativa en la justificación de la reforma, lo cierto es que el cuerpo de la misma dedica mucha más atención a la creación, supresión y fusión de municipios, a las entidades menores y a la colaboración interadministrativa que al detalle de los organismos, entidades, sociedades, fundaciones y demás entes reconducibles a la noción de sector público local, que solo preocupan en tanto que puedan ser un pretexto que impida la estabilidad presupuestaria o la sostenibilidad financiera (BOTO ÁlVAREZ, 2014b).

En este momento, por tanto, solo podemos mirar hacia el futuro con reticencia ya que España continúa con una perspectiva estática y estructural de la organización (PAREJO ALFONSO, 2009, pp. 24-41) y, al menos por ahora, la única preocupación por las fórmulas organizativas públicas es cuantitativa, y reduccionista, y no cualitativa.

\section{BIBLIOGRAFÍA}

Almeida CerReda, M. (2013). La reforma de la planta, estructura competencial, organización y articulación de la Administración local, en La planta del gobierno local (pp. 61-122). Madrid: Fundación Democracia y Gobierno Local.

Álvarez Carreño, S.M. (2009). El proceso de institucionalización de las Agencias Estatales para la mejora de los servicios públicos en España. Via Iuris n. ${ }^{\circ}$ 7, pp. 13-24.

Boto ÁlvareZ, A. (2014a). La inversión francesa en universidad e investigación: la Ley 2013-660 de 22 de julio. Revista General de Derecho Administrativo n. ${ }^{\circ} 35$.

Boto ÁlvareZ, A. (2014b). Redimensionamiento del sector público local, en La reforma del régimen local (pp. 465-518). Valencia: Tirant lo Blanch.

CASSESE, S. (2014). Troppe leggi che restano solo annunci. Corriere della sera. Recuperado el 6 de diciembre de 2014 en http://www.corriere.it/cultura/14_novembre_21/ troppe-leggi-che-restano-solo-annunci-f3a25b44-7145-11e4-b9c7-dbbe3ea603eb. shtml

CONSEIL D'ÉTAT (2001). Les autorités administratives indépendantes - Rapport public. Recuperado el 6 de diciembre de 2014 en http://www.conseil-etat.fr/content/download/368/1132/version/1/file/rapport-public2001.pdf

CORSI, C. (2005). Agenzia e agenzie: una nuova categoria amministrativa? Torino: Giappichelli. 
Cosculluela Montaner, L. (2006). Las Agencias Estatales. Revista Española de Control Externo n. ${ }^{\circ} 24$, pp. 29-53.

Duret, P. (2003), Autorità ed Agenzie e l'Amministrazione in cammino, en Autorità indipendenti e Agenzie. Una ricerca giuridica interdisciplinare. Padova: CEDAM.

Garde Roca, J. A. (Coord.). (2005). La Agencia estatal de evaluación de la calidad y los servicios y de las políticas públicas. Madrid: INAP.

Font i Llovet, T. (2008). Organización administrativa de las Comunidades Autónomas, en Informe Comunidades Autónomas 2007 (pp. 745-754). Barcelona: IDP.

Guettier, C. (2006). Les agences administratives en France. Les Cabiers de la fonction publique et de l'administration 9 (259), pp. 4-10.

MAHERZI, D. (2010). Le développement des agences: un choix risqué? La Revue Administrative 63 (373), pp. 86-95.

Martín-Caro García, F., Bettschen Capa, B. y Estoa Pérez, A. (2007). Las Agencias Estatales. Madrid: Iustel.

Mockle, D. (2006). La gouvernance publique et le droit. Les Cabiers de Droit 47 (1), pp. 89-165.

MoYniHan, D. P. (2006). Ambiguity in policy lessons: the agentification experience. Public Administration 84 (4), pp. 1029-1050.

Napolitano, G. (2014). La logica del diritto amministrativo. Bologna: Il Mulino.

Palomar Olmeda, A. (2010). Reflexiones generales sobre la organización administrativa. Presupuesto y Gasto Público n. ${ }^{\circ}$ 60, pp. 41-55.

Parejo Alfonso, L. (2009). Organización y poder de organización. Madrid: Iustel.

Pérez GÁlveZ, J. F. (2006). El concepto de servicio público en la ley 28/2006, de 18 de julio, sobre agencias estatales. Revista de Derecho 2 (XXVII), Valparaíso. Recuperado el 4 de diciembre de 2014 en http://www.redalyc.org/articulo.oa?id=173620140012

Pérez GÁlvez, J. F. (2007). Las Agencias Estatales para la mejora de los servicios públicos. Granada: Comares.

PiAZZA, S. (1999). Il problema delle "Agenzie" tra crisi del Sistema ministeriale e nuove frome di organizzazione dell'amministrazione. Roma: LUISS.

PollitT, C. et al. (2001). Agency fever? Analysis of an international policy fashion. Journal of Comparative Policy Analysis: Research and Practice n. ${ }^{\circ}$ 3, pp. 271-290. 
PRATS i CATAlÀ, J. (2005). De la burocracia al management, del management a la gobernanza. Las transformaciones de las Administraciones públicas de nuestro tiempo. Madrid: INAP.

Prieto Romero, C. (2010). Gestión directa (I): organismo autónomo local, en Teoría y práctica para la gestión de los servicios locales (pp. 269-336). Madrid: El Consultor-La Ley.

Ramos Carvajal, E. (2013). Un paso más en el proceso de estabilidad presupuestaria y sostenibilidad financiera. Especial incidencia en las Comunidades Autónomas. Revista jurídica de Asturias n. ${ }^{\circ}$ 36, pp. 135-173.

Rodríguez-Arana Muñoz, J. y Sendín García, M. A. (2014). Algunas propuestas para la transposición de la Agencia Estatal a la legislación autonómica. Revista Española de Derecho Administrativo n. ${ }^{\circ} 161$, pp. 19-35.

SAlVAdor ArmendáRIZ, M. A. (2007). Modelo de administración institucional en la Ley de Agencias Estatales para la Mejora de los Servicios Públicos, en Organización y procedimientos administrativos. Libro bomenaje al Profesor Francisco González Navarro (pp. 397-426). Cizur Menor: Aranzadi.

SARAGOSSÀ i SARAGOSSÀ, J. V. (2012). La reorganización institucional de la seguridad social. Revista General de Derecho del Trabajo y de la Seguridad Social n. ${ }^{\circ}$ 29-30.

TACCOGnA, G. (2014). La disciplina dell'organizzazione amministrativa - Lezioni. Lavis: Cedam.

TORIELlO, O. (2014). La riforma della pubblica amministrazione. En: La riforma Renzi della Pubblica Amministrazione (pp. 1-29). Roma: Dike.

Zwart, T. y Verhey, L. (Eds.). (2003). Agencies in European and Comparative Law. AntwerpOxford-New Yord: Intersentia. 